Fl. Medit. 31 (Special Issue): 545-547

https://doi.org/10.7320/FlMedit31SI.545

Version of Record published online on 19 January 2022

\title{
Errata-Corrige. Fl. Medit. 31 (Special Issue)-2021
}

MabBerley, D. J.: The "London" Australian natural history drawings of Ferdinand Bauer (1760-1826) - revisited

Page 69, line 2: "what looked like" should read "what it looked like"

Page 70, line 1: "by using referring" should read "by referring"

Colasante, M. \& AL.: The genus Iris as a critical taxon in establishing an integrated approach to Italian plant biodiversity

Page 230 replace Fig. 15 as follows

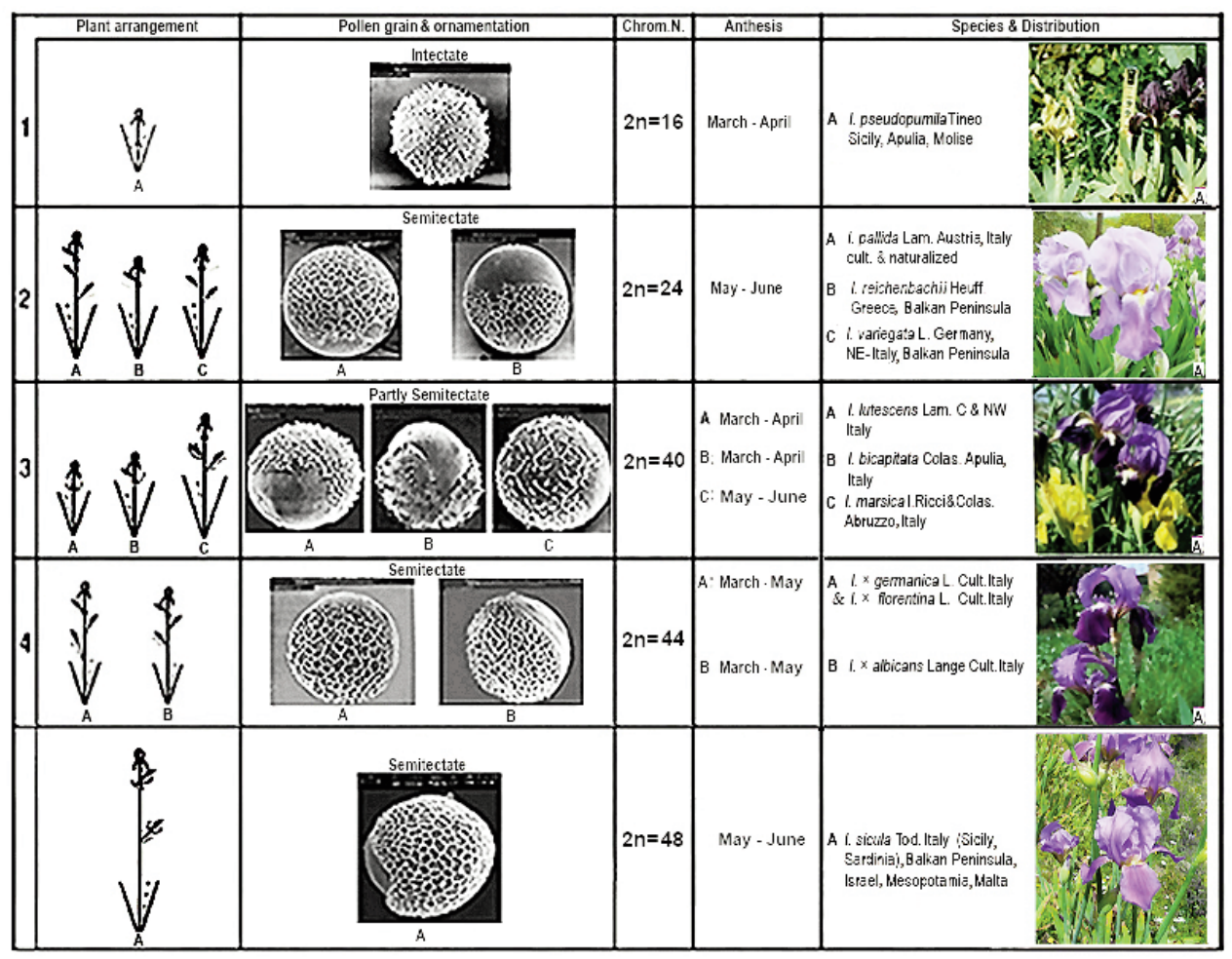


COLDEA, G. \& CRISTEA; V.: Syntaxonomic characterization of the vegetation belts from the

Southeastern Carpathians (Romanian Carpathians)

Page 365, line 11: "Aremonio agrimonioidi" should read "Aremonio agrimonioidis"

PoDDA, L. \& AL.: The vascular flora of the Marine Protected Area of "Capo Carbonara" (SE-Sardinia)

\section{Page 442 replace Fig. $6 \mathrm{~b}$ as follows}

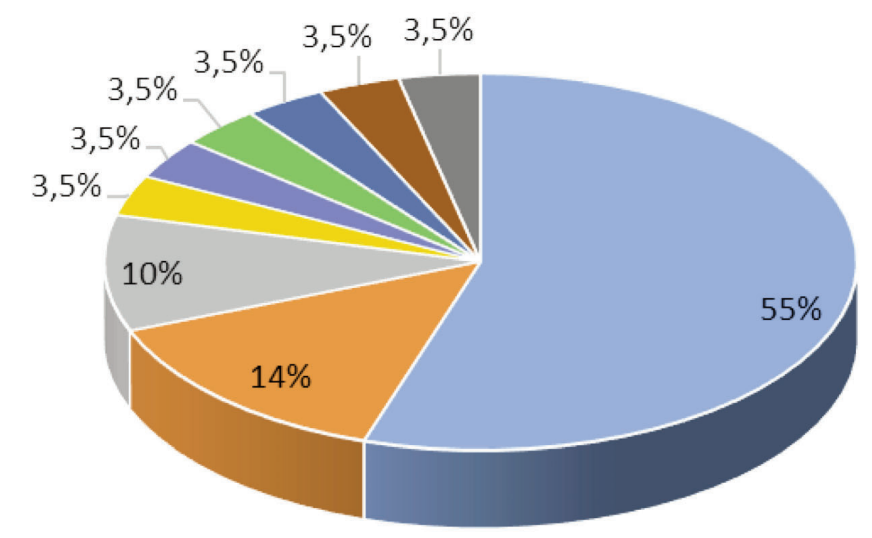

$$
\begin{aligned}
& =\text { SA-CO } \\
& =\text { SA-CO-AT } \\
& =\text { SA } \\
& =\text { SA-SI } \\
& =\text { SA-CO-SI } \\
& =\text { SA-CO-BL } \\
& =\text { SA-CO-SI-TN } \\
& =\text { SA-CO-AT-BL } \\
& =\text { SA-CO-BL-AT-H }
\end{aligned}
$$

BAJONA, E. \& Spadaro, V.: Chorological notes on the Sicilian endemic Euphorbia papillaris (Euphorbiaceae)

Page 465, New site and ecology, line 14: "(1.)" should read "(L.)"

Di Gristina, E. \& Raimondo, F. M.: Muehlenbeckia sagittifolia (Polygonaceae), a new alien for the Italian flora

Page 477, Abstract, line 4: "Muehlenbeckia sagittifolia, native plant" should read "Muehlenbeckia sagittifolia, a native plant"

Page 479, Ecology and uses, line 3: "and medicinal use" should read "and medicinal use (Jankowski \& al. 2000)"

Page 479, legend of Fig. 2: "in the area of Parco della Favorita" should read "in Parco della Favorita"

Pavone, P. \& AL.: New Aloes casual aliens in Sicily

Page 489, Introduction, line 2: "number of infraspecific species and taxa" should read "specific and intraspecific taxa"

Page 489, Introduction, line 4 "in part naturalized" should read "partially naturalized" Page 489, last line "It has recently been reported" should read "It has been recently reported" Page 492, Discussion and Conclusion, line 7 "to also be used as a therapeutic" should read "to also be consumed as a therapeutic remedy" 
Piccione, V. \& Malacrinò, V.: Anredera cordifolia (Basellaceae) invasive in the river vegetation of North-Eastern Sicily)

Page 502, legend of Fig. 1: "Arendera" should read "Anredera"

Page 503, legend of Fig. 2: "Arendera" should read "Anredera"

LASEN, C.: Biodiversité végétale, valeurs naturelles et sauvegarde du paysage dans le domaine dolomitique

Page 524, line 25: "qui tienne edonnée.sn compte" should read "qui tienne en compte"

Page 527, sixth line from last: "de type azonale" should read "de type azonal"

Page 532, thirteenth line from last: "etc.)." should read "etc."

Page 533, sixth line from last: "quelques éléments" should read "quelque élément"

Page 539, line 1 after Bibliographie: "plaques photographiques" should read "planches photographiques"

Page 539, line 3 after Bibliographie: "plaques photographiques" should read "planches photographiques"

Page 540, line 1: "PLAQUES PHOTOGRAPHIQUES" should read "PLANCHES PHOTOGRAPHIQUES"

Page 540, caption of Figure: "Plaque 1" should read "Planche 1"

Page 541, caption of Figure: "Plaque 2" should read "Planche 2"

Page 542, caption of Figure: "Plaque 3" should read "Planche 3"

Page 543, caption of Figure: "Plaque 4" should read "Planche 4"

Page 544, caption of Figure: "Plaque 5" should read "Planche 5" 